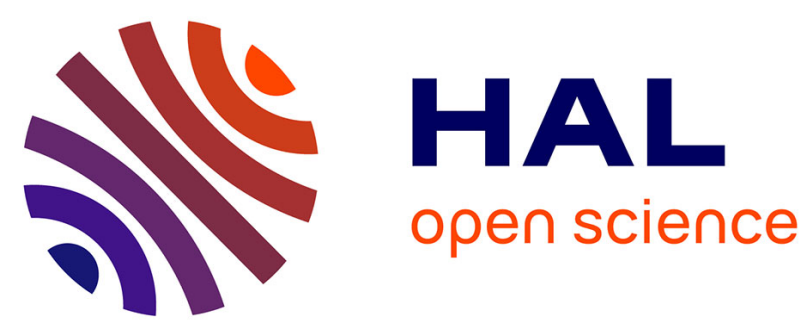

\title{
On Fixed-Time Interval Estimation of Discrete-Time Nonlinear Time-Varying Systems With Disturbances
}

\author{
Thach Ngoc Dinh, Frédéric Mazenc, Zhenhua Wang, Tarek Raissi
}

\section{To cite this version:}

Thach Ngoc Dinh, Frédéric Mazenc, Zhenhua Wang, Tarek Raissi. On Fixed-Time Interval Estimation of Discrete-Time Nonlinear Time-Varying Systems With Disturbances. ACC 2020 - American Control Conference, Jul 2020, Denver, CO, United States. 10.23919/ACC45564.2020.9147754 . hal$03113667 \mathrm{v} 2$

\section{HAL Id: hal-03113667 \\ https://hal-cnam.archives-ouvertes.fr/hal-03113667v2}

Submitted on 28 Feb 2020

HAL is a multi-disciplinary open access archive for the deposit and dissemination of scientific research documents, whether they are published or not. The documents may come from teaching and research institutions in France or abroad, or from public or private research centers.
L'archive ouverte pluridisciplinaire HAL, est destinée au dépôt et à la diffusion de documents scientifiques de niveau recherche, publiés ou non, émanant des établissements d'enseignement et de recherche français ou étrangers, des laboratoires publics ou privés. 


\title{
On Fixed-Time Interval Estimation of Discrete-Time Nonlinear Time-Varying Systems With Disturbances
}

\author{
Thach Ngoc Dinh ${ }^{1}$, Frederic Mazenc ${ }^{2}$, Zhenhua Wang ${ }^{3}$ and Tarek Raïssi ${ }^{1}$
}

\begin{abstract}
The aim of this paper is to cope with estimation issues of discrete-time nonlinear time-varying systems with input and output. Inspired by [12], a new design technique of fixed-time observers is proposed. It relies on the use of past values of the output and the theory of the monotone systems to construct dead bit observer or fixed-time interval estimator depending on the absence or the presence of uncertainties. Finally, simulations are conducted to verify the effectiveness of the proposed schemes.
\end{abstract}

\section{INTRODUCTION}

It is known that state estimation is important in control theory and applications. Various state estimation methods are available in the literature [8], [1]. To improve the accuracy, many robust methods have been proposed to attenuate the effect of ubiquitous uncertainties. Kalman filtering and $H_{\infty}$ observer/filter design are two commonly used robust estimation techniques. The uncertainties in Kalman filtering are assumed to be Gaussian noises while the $H_{\infty}$ design methods assume that the uncertainties are energy-bounded. Note that both Gaussian noise assumption and energybounded assumption are impractical. A general and practical assumption is that the uncertainties are unknown but bounded by known bounds. With this assumption, interval estimation has attracted much attention in the past decades [7], [14].

In the literature, there are mainly two kinds of interval estimation methods: one is interval estimation based on geometrical sets and the other uses interval observers. Compared with the interval estimation methods based on geometrical sets, the interval observer is easy to implement with high computation efficiency. The basic idea of interval observer designs is to construct stable and cooperative error dynamics. However, it is non-trivial to construct such an error system. To deal with this difficulty, coordinate transformation-based interval observer design methods have been proposed to relax the design conditions [11], [5]. Although the coordinate transformation-based methods can relax design conditions and broaden application scope, the interval estimation results may be very conservative. Different to the coordinate transformation-based methods, [16] proposes a new interval observer with more degrees of design freedom and presented

\footnotetext{
${ }^{1}$ Thach Ngoc Dinh and Tarek Raïssi are with Conservatoire National des Arts et Métiers (CNAM), Cedric-Lab, 292 rue St-Martin, 75141 Paris Cedex 03, France ngoc-thach. dinhelecnam.net, tarek.raissi@cnam.fr

${ }^{2}$ Frederic Mazenc is with EPI DISCO Inria-Saclay, L2S, CNRS CentraleSupélec, 3 rue Joliot Curie, 91192 Gif-sur-Yvette, France frederic.mazencel2s.centralesupelec.fr

${ }^{3}$ Zhenhua Wang is with School of Astronautics, Harbin Institute of Technology, West Da-Zhi Street, 150001 Harbin, PR China zhenhua.wang@hit.edu.cn
}

a robust design. The result proposed in [16] can be optimized to improve estimation accuracy.

Note that the aforemetioned methods only consider the stability and robustness of the interval estimation. In some situations, we are interested in achieving some transient performance, e.g., guaranteeing the fixed-time convergence of the estimation error. A fixed-time observer was first proposed and designed in [6]. Following this pioneering work, some works have been done on fixed-time observer design [9], [13], [15]. However, most existing results on fixed-time observer focus on continuous-time systems. To the best of our knowledge, only [17], [3], [4] consider fixed-time observer for discrete-time systems, but they do not design the interval estimation or do not study the time-varying context. In this paper, based on formulas incorporating past values of the input and the output of the studied plant, two goals can be achieved. In the absence of unknown uncertainties and after a fixed time, the exact values of the solutions are obtained. Next when unknown disturbances are present and are upper and lower bounded by known constant vectors, after a fixed time, the formulas we exhibit provide upper and lower bounds for each component of the solutions, as interval observers do.

The paper is organized as follows. Preliminaries and problem setup are introduced in Section II. Dead bit estimation of the solutions in the absence of uncertainties are given in Section III. Intervals for the solutions in the presence of uncertainties are designed in Section IV. An example illustrates the main results in Section V. Concluding remarks are given in Section VI.

\section{PRELIMINARIES AND PROBLEM SETUP}

\section{A. Basic notations, definitions and properties}

A matrix $Q \in \mathbb{R}^{n \times n}$ is called Schur stable if the norm of all its eigenvalues is smaller than one. The identity matrix of any dimension is denoted by $I$. The Euclidean norm of a vector $x \in \mathbb{R}^{n}$ is denoted by $|x|$. Inequalities must be understood component-wise, i.e., for $x_{a}=\left[x_{a, 1}, \ldots, x_{a, n}\right]^{\top} \in$ $\mathbb{R}^{n}$ and $x_{b}=\left[x_{b, 1}, \ldots, x_{b, n}\right]^{\top} \in \mathbb{R}^{n}, x_{a} \leq x_{b}$ if and only if, for all $i \in\{1, \ldots, n\}, x_{a, i} \leq x_{b, i}$. For a square matrix $Q \in \mathbb{R}^{n \times n}$, the determinant of $Q$ is $\operatorname{denoted} \operatorname{det}(Q)$ and we define $Q^{+}, Q^{-} \in \mathbb{R}^{n \times n}$ by $Q^{+}=\max (Q, 0)$ and $Q^{-}=$ $Q^{+}-Q$.

Definition 1: For any undriven response as follows

$$
x(k+1)=M(k) x(k), k \in \mathbb{N},
$$

the discrete-time state transition matrix is a term which relates the state of (1) at time $k$ to the state at an earlier 
time $\ell \in \mathbb{N}$ :

$$
x(k)=\Phi_{M}(k, \ell) x(\ell), k \geq \ell,
$$

where

$$
\Phi_{M}(k, \ell)=\left\{\begin{array}{l}
M(k-1) M(k-2) \cdots M(\ell), k>\ell \geq 0, \\
I, k=\ell
\end{array}\right.
$$

Remark 1: When $M(k-1), M(k-2), \cdots, M(\ell)$ are all invertible, then

- $\Phi_{M}(k, \ell)$ is nonsingular and

$$
\Phi_{M}^{-1}(k, \ell)=\left\{\begin{array}{lr}
M^{-1}(\ell) M^{-1}(\ell+1) \cdots M^{-1}(k-1), \\
I, k=\ell . & k>\ell \geq 0,
\end{array}\right.
$$

Then, one can define $\Phi_{M}(\ell, k)$ when $\ell<k$ by

$$
\Phi_{M}(\ell, k)=\Phi_{M}^{-1}(k, \ell) .
$$

- For all $k, \ell, m$ in $\mathbb{N}$, the next well-known property of the discrete-time state transition matrix can be easily deduced and will be frequently used throughout the paper

$$
\Phi_{M}(k, m) \Phi_{M}(m, \ell)=\Phi_{M}(k, \ell) .
$$

\section{B. Family of studied systems}

Consider the following discrete-time nonlinear timevarying system:

$\left\{\begin{array}{l}x(k+1)=A(k) x(k)+\beta(k, u(k), y(k))+d(k) \\ y(k)=C(k) x(k)+v(k)\end{array} \quad k \in \mathbb{N}\right.$.

where $x(k) \in \mathbb{R}^{n}$ is the state, $\beta$ is a nonlinear function, $A(k): \mathbb{N} \rightarrow \mathbb{R}^{n \times n}$ and $C(k): \mathbb{N} \rightarrow \mathbb{R}^{q \times n}, y(k) \in \mathbb{R}^{q}$ is the output, $u(k) \in \mathbb{R}^{p}$ is an input, and $d: \mathbb{N} \rightarrow \mathbb{R}^{n}, v: \mathbb{N} \rightarrow$ $\mathbb{R}^{q}$ are respectively additive disturbances and measurement noises.

Goal and Method. In this note, two objectives are simultaneously achieved for the discrete-time system (7): (i) a dead bit of the state when the functions $d$ and $v$ are known and (ii) a fixed-time interval estimation when the functions $d$ and $v$ are unknown but bounded by known values. It is worth highlighting that the design of two bounds (i.e., the interval) for the solutions in fixed time can be done without requesting an appropriate knowledge of the interval of the initial conditions and a direct or indirect notion of nonnegative and cooperative system like in many other works on interval observer designs of discrete-time systems [2], [10].

The following assumptions are introduced:

Assumption 1: For all $k \in \mathbb{N}$, the pair $(A(k), C(k))$ is observable and $A(k)$ is invertible. Furthermore, there exists $L(k): \mathbb{N} \rightarrow \mathbb{R}^{n \times q}$ such that the matrix $H(k)=A(k)+$ $L(k) C(k)$ is both Schur stable and invertible.

Assumption 2: There are known constant vectors $\underline{d} \in$ $\mathbb{R}^{n}, \bar{d} \in \mathbb{R}^{n}$ and $\underline{v} \in \mathbb{R}^{q}, \bar{v} \in \mathbb{R}^{q}$ such that for all $k \in \mathbb{N}$, the inequalities

$$
\begin{aligned}
& \underline{d} \leq d(k) \leq \bar{d} \\
& \underline{v} \leq v(k) \leq \bar{v}
\end{aligned}
$$

are satisfied.

\section{Discussion of the Assumptions:}

- The fact that for all $k \in \mathbb{N}$, the pair $(A(k), C(k))$ observable implies that there is a matrix $L(k) \in \mathbb{R}^{n \times q}$ such that the matrix

$$
H(k)=A(k)+L(k) C(k) \in \mathbb{R}^{n \times n}
$$

Schur stable is rather standard in the context of the design of observers [5].

- Assuming that $A(k)$ is invertible is not restrictive at all because when $(A(k), C(k))$ is observable and $A(k)$ is not invertible, we can always decompose $A(k) x+$ $\beta(k, u(k), y(k))$ in an alternative way so that the new matrix $A(k)$ is invertible. Similarly, assuming that $H(k)$ is invertible is reasonable because we can always choose $L(k)$ so that $H(k)$ is both Schur stable and invertible.

- Assumption 2 is realistic: it is frequently satisfied in practice. It can be relaxed by allowing the bounds to depend on time $k$ but for the sake of the simplicity, we restrict ourselves to the case where they are constant.

\section{DeAd Bit Estimation of the STATe}

The technique proposed in this section allows a dead bit estimation of the state, but it can be applied only when the functions $d$ and $v$ are known. Let us state and prove the following result:

Theorem 1: Let the system (7) satisfy Assumptions 1. Let $L(k) \in \mathbb{R}^{n \times q}$ and $h \in \mathbb{N}, h \geq 1$ be such that the matrix $\Phi_{H}^{-1}(k, k-h)-\Phi_{A}^{-1}(k, k-h)$ is invertible for all $k \in \mathbb{N}$, where $\Phi_{H}^{-1}(k, k-h)$ and $\Phi_{A}^{-1}(k, k-h)$ defined in (4) are the inverses of discrete-time transition matrices $\Phi_{H}(k, k-h)$ and $\Phi_{A}(k, k-h)$, respectively. Then, for a given input $u(k)$, any solution $x(k)$ of the system (7) which exists over $\mathbb{N}$ satisfies, for all $k \geq h$,

$$
\begin{aligned}
x(k)= & -E_{h}(k) \sum_{\ell=k-h}^{k-1} \Phi_{A}(k-h, \ell+1) \beta(\ell, u(\ell), y(\ell)) \\
& +E_{h}(k) \sum_{\ell=k-h}^{k-1} \Phi_{H}(k-h, \ell+1)[\beta(\ell, u(\ell), y(\ell)) \\
& \left.-E_{h}(k) \sum_{\ell=k-h}^{k-1} \Phi_{A}(k-h, \ell+1) d(\ell)\right] \\
& +E_{h}(k) \sum_{\ell=k-h}^{k-1} \Phi_{H}(k-h, \ell+1)(d(\ell)+L(\ell) v(\ell)),
\end{aligned}
$$

with

$$
E_{h}(k)=\left(\Phi_{H}^{-1}(k, k-h)-\Phi_{A}^{-1}(k, k-h)\right)^{-1} .
$$

Proof: The matrices $A(k)$ and $H(k)$ are invertible for all $k \in \mathbb{N}$. It follows that $\Phi_{A}(k, k-h)$ and $\Phi_{H}(k, k-h)$ are invertible for all $k \in \mathbb{N}, h \geq 1$ (see Remark 1). From 
the definition of the output $y$ and the definition of $H(k)$, we deduce that the system (7) admits two representations:

$$
\begin{aligned}
x(k+1)= & A(k) x(k)+\beta(k, u(k), y(k))+d(k), \\
x(k+1)= & H(k) x(k)+\beta(k, u(k), y(k)) \\
& -L(k) y(k)+d(k)+L(k) v(k) .
\end{aligned}
$$

By combining all equations of these two systems between two values $m_{1} \in \mathbb{N}, m_{2} \in \mathbb{N}$ and $m_{1} \geq m_{2}$, we have

$$
\begin{aligned}
x\left(m_{1}\right)=\Phi_{A}\left(m_{1}, m_{2}\right) x\left(m_{2}\right)+ & \sum_{\ell=m_{2}}^{m_{1}-1} \Phi_{A}\left(m_{1}, \ell+1\right) \times \\
& \times[\beta(\ell, u(\ell), y(\ell))+d(\ell)],
\end{aligned}
$$

$$
\begin{aligned}
x\left(m_{1}\right)= & \Phi_{H}\left(m_{1}, m_{2}\right) x\left(m_{2}\right)+\sum_{\ell=m_{2}}^{m_{1}-1} \Phi_{H}\left(m_{1}, \ell+1\right) \times \\
& \times[\beta(\ell, u(\ell), y(\ell))-L(\ell) y(\ell)+d(\ell)+L(\ell) v(\ell)] .
\end{aligned}
$$

Now, consider a value $k \geq h$. Then selecting $m_{2}=k-h$ and $m_{1}=k$, the equalities (15)-(16) give

$$
\begin{aligned}
x(k)= & \Phi_{A}(k, k-h) x(k-h)+\sum_{\ell=k-h}^{k-1} \Phi_{A}(k, \ell+1) \times \\
& \times[\beta(\ell, u(\ell), y(\ell))+d(\ell)], \\
x(k)= & \Phi_{H}(k, k-h) x(k-h)+\sum_{\ell=k-h}^{k-1} \Phi_{H}(k, \ell+1) \times \\
& \times[\beta(\ell, u(\ell), y(\ell))-L(\ell) y(\ell)+d(\ell)+L(\ell) v(\ell)] .
\end{aligned}
$$

Bearing in mind the properties (5)-(6) of the state transition matrices, we obtain immediately:

$$
\begin{aligned}
& \left(\Phi_{H}^{-1}(k, k-h)-\Phi_{A}^{-1}(k, k-h)\right) x(k) \\
& =-\sum_{\ell=k-h}^{k-1} \Phi_{A}(k-h, \ell+1)[\beta(\ell, u(\ell), y(\ell))+d(\ell)] \\
& +\sum_{\ell=k-h}^{k-1} \Phi_{H}(k-h, \ell+1)[\beta(\ell, u(\ell), y(\ell))-L(\ell) y(\ell) \\
& +d(\ell)+L(\ell) v(\ell)] .
\end{aligned}
$$

Since $\Phi_{H}^{-1}(k, k-h)-\Phi_{A}^{-1}(k, k-h)$ is assumed to be invertible, we deduce that (11) is satisfied.

Remark 2: Because the pair $(A(k), C(k))$ is observable for all $k \in \mathbb{N}$, there are matrices $L(k), L(k-1) \ldots$, $L(k-h)$ and $h_{*} \in \mathbb{N}$ such that for all $h \in \mathbb{N}, h \geq h_{*}$, $\left|\Phi_{H}(h, k-h)\right|\left|\Phi_{A}^{-1}(h, k-h)\right|<1$. Consequently the fact that $\Phi_{H}^{-1}(k, k-h)-\Phi_{A}^{-1}(k, k-h)$ invertible is not restrictive.

The formula (11) may contain many terms because $h$ may be large and thus many values have to be stored. To overcome this drawback, we propose an alternative solution which is based on dynamic extensions.
Theorem 2: Let the system (7) and $L(k)$ satisfy the Assumption 1, let $h$ be defined as in Theorem 1 and let $u$ be a given input. Consider the dynamic extensions

$$
\hat{x}(k+1)=A(k) \hat{x}(k)+\beta(k, u(k), y(k))+d(k)
$$

and

$$
\begin{aligned}
x_{*}(k+1)= & H(k) x_{*}(k)+\beta(k, u(k), y(k)) \\
& -L(k) y(k)+d(k)+L(k) v(k) .
\end{aligned}
$$

Consider a solution $x(k)$ of (7) defined over $\mathbb{N}$. Then, for all $k \geq h$,

$$
\begin{aligned}
x(k)=E_{h}(k)[ & \Phi_{H}^{-1}(k, k-h) x_{*}(k)-x_{*}(k-h) \\
& \left.-\Phi_{A}^{-1}(k, k-h) \hat{x}(k)+\hat{x}(k-h)\right] .
\end{aligned}
$$

Remark 3: Notice that (21) is a classical observer for the system (7) when disturbances are known. Moreover, it is always possible to choose a decomposition of $\beta(k, u(k), y(k))$ and a gain $L(k)$ so that the corresponding matrices $A(k)$ and $H(k)$ are Schur stable, thus (20) and (21) are stable.

Proof: Consider a solution $\left(\hat{x}(k), x_{*}(k)\right)$ of (20)-(21) associated with the solution $x(k)$ defined over $\mathbb{N}$. Then, arguing as we did in the proof of Theorem 1, we have

$$
\begin{aligned}
\hat{x}(k)=\Phi_{A}(k, k-h) \hat{x}(k-h) & +\sum_{\ell=k-h}^{k-1} \Phi_{A}(k, \ell+1) \times \\
& \times[\beta(\ell, u(\ell), y(\ell))+d(\ell)],
\end{aligned}
$$

$$
\begin{aligned}
x_{*}(k)= & \Phi_{H}(k, k-h) x_{*}(k-h)+\sum_{\ell=k-h}^{k-1} \Phi_{H}(k, \ell+1) \times \\
& \times[\beta(\ell, u(\ell), y(\ell))-L(\ell) y(\ell)+d(\ell)+L(\ell) v(\ell)] .
\end{aligned}
$$

From properties (5)-(6), it follows that for all $k \geq h$,

$$
\begin{aligned}
& \sum_{\ell=k-h}^{k-1} \Phi_{A}(k-h, \ell+1)[\beta(\ell, u(\ell), y(\ell))+d(\ell)] \\
& =\Phi_{A}^{-1}(k, k-h) \hat{x}(k)-\hat{x}(k-h), \\
& \sum_{\ell=k-h}^{k-1} \Phi_{H}(k-h, \ell+1)[\beta(\ell, u(\ell), y(\ell)) \\
& \quad-L(\ell) y(\ell)+d(\ell)+L(\ell) v(\ell)] \\
& =\Phi_{H}^{-1}(k, k-h) x_{*}(k)-x_{*}(k-h) .
\end{aligned}
$$

Combining (11), (25) and (26), we obtain, for all $k \geq h$,

$$
\begin{aligned}
E_{h}^{-1}(k) x(k)= & \Phi_{H}^{-1}(k, k-h) x_{*}(k)-x_{*}(k-h) \\
& -\Phi_{A}^{-1}(k, k-h) \hat{x}(k)+\hat{x}(k-h) .
\end{aligned}
$$

This allows us to conclude.

\section{FiXed-time Interval estimation}

Theorems 1 and 2 give in fixed time the exact value of any solution $x(k)$ of (7). However, these estimations cannot be used when the disturbances $d$ and $v$ are unknown. The second objective of the present note is to overcome this limitation by assuming only that bounds $\underline{d}, \bar{d}$ and $\underline{v}, \bar{v}$ are known. 
In this section, we consider the case where Assumptions 1 and 2 are satisfied and the matrix $L(k)$ is selected as described in (10). Next, we introduce the following matrices

$$
\begin{aligned}
& F_{h}(k)=-E_{h}(k) \Phi_{A}^{-1}(k, k-h), \\
& G_{h}(k)=E_{h}(k) \Phi_{H}^{-1}(k, k-h) .
\end{aligned}
$$

and the vectors

$$
\begin{aligned}
& d_{L}(k)=\left(\sum_{\ell=k-h}^{k-1} F_{h}(k) \Phi_{A}(k, \ell+1)\right. \\
& \left.+G_{h}(k) \Phi_{H}(k, \ell+1)\right)^{+} \bar{d} \\
& -\left(\sum_{\ell=k-h}^{k-1} F_{h}(k) \Phi_{A}(k, \ell+1)\right. \\
& \left.+G_{h}(k) \Phi_{H}(k, \ell+1)\right)^{-} \underline{d} \\
& d_{S}(k)=\left(\sum_{\ell=k-h}^{k-1} F_{h}(k) \Phi_{A}(k, \ell+1)\right. \\
& \left.+G_{h}(k) \Phi_{H}(k, \ell+1)\right)^{+} \underline{d} \\
& -\left(\sum_{\ell=k-h}^{k-1} F_{h}(k) \Phi_{A}(k, \ell+1)\right. \\
& \left.+G_{h}(k) \Phi_{H}(k, \ell+1)\right)^{-} \bar{d} \\
& v_{L}(k)=\left(G_{h}(k) \sum_{\ell=k-h}^{k-1} \Phi_{H}(k, \ell+1) L\right)^{+} \bar{v} \\
& -\left(G_{h}(k) \sum_{\ell=k-h}^{k-1} \Phi_{H}(k, \ell+1) L\right)^{-} \underline{v}, \\
& v_{S}(k)=\left(G_{h}(k) \sum_{\ell=k-h}^{k-1} \Phi_{H}(k, \ell+1) L\right)^{+} \underline{v} \\
& -\left(G_{h}(k) \sum_{\ell=k-h}^{k-1} \Phi_{H}(k, \ell+1) L\right)^{-} \bar{v} .
\end{aligned}
$$

We are ready to state and prove the following result:

Theorem 3: Let the system (7) satisfy Assumptions 1 and 2 and let $L(k)$ and $h \in \mathbb{N}, h \geq 1$ be such that $E_{h}(k)$ given in (12) is well-defined. Let $u$ be a given input and consider a solution $x(k)$ of the system (7) defined over $\mathbb{N}$. Then, for all integer $k \geq h$, the inequalities

$$
\underline{x}(k) \leq x(k) \leq \bar{x}(k) .
$$

with

$$
\begin{aligned}
\bar{x}(k)= & -E_{h}(k) \sum_{\ell=k-h}^{k-1} \Phi_{A}(k-h, \ell+1) \beta(\ell, u(\ell), y(\ell)) \\
& +E_{h}(k) \sum_{\ell=k-h}^{k-1} \Phi_{H}(k-h, \ell+1)[\beta(\ell, u(\ell), y(\ell)) \\
& \left.+d_{L}(k)+v_{L}(k), \quad-L(\ell) y(\ell)\right] \\
\bar{x}(k)= & -E_{h}(k) \sum_{\ell=k-h}^{k-1} \Phi_{A}(k-h, \ell+1) \beta(\ell, u(\ell), y(\ell)) \\
& +E_{h}(k) \sum_{\ell=k-h}^{k-1} \Phi_{H}(k-h, \ell+1)[\beta(\ell, u(\ell), y(\ell)) \\
& +d_{S}(k)+v_{S}(k),
\end{aligned}
$$

hold.

Proof: From (11), we have

$$
\begin{aligned}
x(k)= & -E_{h}(k) \sum_{\ell=k-h}^{k-1} \Phi_{A}(k-h, \ell+1) \beta(\ell, u(\ell), y(\ell)) \\
& +E_{h}(k) \sum_{\ell=k-h}^{k-1} \Phi_{H}(k-h, \ell+1)[\beta(\ell, u(\ell), y(\ell)) \\
& -E_{h}(k) \sum_{\ell=k-h}^{k-1} \Phi_{A}(k-h, \ell+1) d(\ell) \\
& +E_{h}(k) \sum_{\ell=k-h}^{k-1} \Phi_{H}(k-h, \ell+1)(d(\ell)+L(\ell) v(\ell)) .
\end{aligned}
$$

From (37) and the definition of $F_{h}(k)$ and $G_{h}(k)$ in (28)(29), it follows that, for all $k \geq h$,

$$
\begin{gathered}
x(k)=-E_{h}(k) \sum_{\ell=k-h}^{k-1} \Phi_{A}(k-h, \ell+1) \beta(\ell, u(\ell), y(\ell)) \\
+E_{h}(k) \sum_{\ell=k-h}^{k-1} \Phi_{H}(k-h, \ell+1)[\beta(\ell, u(\ell), y(\ell)) \\
-L(\ell) y(\ell)] \\
+\sum_{\ell=k-h}^{k-1}\left(F_{h}(k) \Phi_{A}(k, \ell+1)+G_{h}(k) \Phi_{H}(k, \ell+1)\right) d(\ell) \\
+G_{h}(k) \sum_{\ell=k-h}^{k-1} \Phi_{H}(k, \ell+1) L v(\ell) .
\end{gathered}
$$


From Assumption 2, we deduce that, for all $k \geq h$,

$$
\begin{aligned}
& \left(\sum_{\ell=k-h}^{k-1} F_{h}(k) \Phi_{A}(k, \ell+1)+G_{h}(k) \Phi_{H}(k, \ell+1)\right)^{+} \underline{d} \\
& -\left(\sum_{\ell=k-h}^{k-1} F_{h}(k) \Phi_{A}(k, \ell+1)+G_{h}(k) \Phi_{H}(k, \ell+1)\right)^{-} \bar{d} \\
& \leq \sum_{\ell=k-h}^{k-1}\left(F_{h}(k) \Phi_{A}(k, \ell+1)+G_{h}(k) \Phi_{H}(k, \ell+1)\right) d(\ell) \\
& \leq\left(\sum_{\ell=k-h}^{k-1} F_{h}(k) \Phi_{A}(k, \ell+1)+G_{h}(k) \Phi_{H}(k, \ell+1)\right)^{+} \bar{d} \\
& -\left(\sum_{\ell=k-h}^{k-1} F_{h}(k) \Phi_{A}(k, \ell+1)+G_{h}(k) \Phi_{H}(k, \ell+1)\right)^{-} \underline{d}
\end{aligned}
$$

Similarly,

$$
\begin{aligned}
& \left(G_{h}(k) \sum_{\ell=k-h}^{k-1} \Phi_{H}(k, \ell+1) L\right)^{+} \underline{v} \\
& -\left(G_{h}(k) \sum_{\ell=k-h}^{k-1} \Phi_{H}(k, \ell+1) L\right)^{-} \bar{v} \\
& \leq G_{h}(k) \sum_{\ell=k-h}^{k-1} \Phi_{H}(k, \ell+1) L v(\ell) \\
& \leq\left(G_{h}(k) \sum_{\ell=k-h}^{k-1} \Phi_{H}(k, \ell+1) L\right)^{+} \bar{v} \\
& \quad-\left(G_{h}(k) \sum_{\ell=k-h}^{k-1} \Phi_{H}(k, \ell+1) L\right)^{-} \underline{v} .
\end{aligned}
$$

It follows that

$$
\begin{aligned}
& d_{S}(k) \\
& \leq \sum_{\ell=k-h}^{k-1}\left(F_{h}(k) \Phi_{A}(k, \ell+1)+G_{h}(k) \Phi_{H}(k, \ell+1)\right) d(\ell) \\
& \leq d_{L}(k), \\
& v_{S}(k) \leq G_{h}(k) \sum_{\ell=k-h}^{k-1} \Phi_{H}(k, \ell+1) L v(\ell) \leq v_{L}(k)
\end{aligned}
$$

with $d_{L}(k), d_{S}(k), v_{L}(k), v_{S}(k)$ are the vectors defined in (30), (31), (32), (33).

From (38), (39) and (40), we can conclude.

The motivations of Theorem 2 also motivate the following result:

Theorem 4: Let the system (7) satisfy the conditions in Theorem 3. Let $u(k)$ be a given input and consider a solution of (7) defined over $\mathbb{N}$. Let us introduce dynamic extensions:

$$
\begin{aligned}
& z_{a}(k+1)=A(k) z_{a}(k)+\beta(k, u(k), y(k)), \\
& z_{h}(k+1)=H(k) z_{h}(k)+\beta(k, u(k), y(k))-L(k) y(k),
\end{aligned}
$$

Then, for all $k \geq h$, the following inequalities are satisfied:

$$
\underline{\Upsilon}\left(Z_{k}\right) \leq x(k) \leq \bar{\Upsilon}\left(Z_{k}\right),
$$

with $Z=\left(z_{a}, z_{h}\right)$ and the bounds $\bar{\Upsilon}, \underline{\Upsilon}$ are an estimated interval for the system (7) given by

$$
\begin{aligned}
& \bar{\Upsilon}\left(Z_{k}\right)=E_{h}(k)\left[z_{a}(k-h)-\Phi_{A}^{-1}(k, k-h) z_{a}(k)\right. \\
& \left.+\Phi_{H}^{-1}(k, k-h) z_{h}(k)-z_{h}(k-h)\right]+d_{L}(k)+v_{L}(k) \\
& \bar{\Upsilon}\left(Z_{k}\right)=E_{h}(k)\left[z_{a}(k-h)-\Phi_{A}^{-1}(k, k-h) z_{a}(k)\right. \\
& \left.+\Phi_{H}^{-1}(k, k-h) z_{h}(k)-z_{h}(k-h)\right]+d_{S}(k)+v_{S}(k),
\end{aligned}
$$

where $d_{L}(k), d_{S}(k), v_{L}(k), v_{S}(k)$ are the vectors defined in (30), (31), (32), (33).

Proof: For a solution $x(k)$ of (7) defined over $\mathbb{N}$, we have

$$
\begin{aligned}
z_{a}(k) & =\Phi_{A}(k, k-h) z_{a}(k-h) \\
& +\sum_{\ell=k-h}^{k-1} \Phi_{A}(k, \ell+1) \beta(\ell, u(\ell), y(\ell)) \\
z_{h}(k) & =\Phi_{H}(k, k-h) z_{h}(k-h) \\
& +\sum_{\ell=k-h}^{k-1} \Phi_{H}(k, \ell+1)[\beta(\ell, u(\ell), y(\ell))-L(\ell) y(\ell)] .
\end{aligned}
$$

These equalities can be rewritten as

$$
\begin{aligned}
-\sum_{\ell=k-h}^{k-1} \Phi_{A}(k-h, & \ell+1) \beta(\ell, u(\ell), y(\ell)) \\
& =z_{a}(k-h)-\Phi_{A}^{-1}(k, k-h) z_{a}(k),
\end{aligned}
$$

$$
\begin{aligned}
\sum_{\ell=k-h}^{k-1} \Phi_{H}(k-h, \ell & +1)[\beta(\ell, u(\ell), y(\ell))-L(\ell) y(\ell)] \\
& =\Phi_{H}^{-1}(k, k-h) z_{h}(k)-z_{h}(k-h) .
\end{aligned}
$$

Theorem 3 ensures that the inequalites (34) hold. Then from these inequalities and the equalities (46) and (47), we deduce that the inequalities (43) are satisfied.

\section{AN EXAMPLE}

In this section, we consider the system borrowed from [18] which is of the form (7) with $C=[-0.51 .50]$,

$$
\begin{aligned}
& A(k)=\left[\begin{array}{rrr}
0.2 e^{-\frac{k}{100}} & 0.6 & 0 \\
0 & 0.5 & \sin (k) \\
0 & 0 & 0.7
\end{array}\right], \\
& \beta(u(k), y(k))=\left[\begin{array}{c}
0.2 \sin (0.2 k) \\
1.8 \sin (0.2 k) \\
0.3 \sin (0.2 k)
\end{array}\right], \\
& d(k)=\left[\begin{array}{c}
\frac{1}{4} \sin (2 k) \\
\frac{1}{4} \sin (2 k) \\
\frac{1}{4} \sin (2 k)
\end{array}\right] \text { and } v(k)=0.3 \cos (k) .
\end{aligned}
$$


We choose $L(k)=\left[\begin{array}{lll}\frac{1}{4} & 0 & 0\end{array}\right]^{\top}$. When $h=\{3 ; 4\}$, one can check by Matlab that $\operatorname{det}(A(k))$ and $\operatorname{det}(A(k)+L(k) C(k))$ are different from 0 for all $k \in \mathbb{N}$. That means that the matrices $A(k)$ and $H(k)$ are invertible for all $k \in \mathbb{N}$. Then, all assumptions are satisfied for $h=\{3 ; 4\}$.

We apply Theorem 2 and select the initial values $x_{1}(0)=$ $1, x_{2}(0)=-1$ and $x_{3}(0)=2$. Then, for the value of the delay $h=3$, we implement the dead bit estimation of the state $x$ given by (22). The simulation result is plotted in Fig. 1.

Finally, we implement the dynamic extensions $x_{a}$ and $x_{h}$ given by (41)-(42), and the upper and lower bounds given by (44)-(45). Fig. 2 illustrates one example where $h=4$ with the same initial values and input. We choose the known bounds of disturbances $\bar{d}=\left[\frac{1}{4} \frac{1}{4} \frac{1}{4}\right]^{\top}, \underline{d}=-\bar{d}, \bar{v}=0.3$ and $\underline{v}=-0.3$.
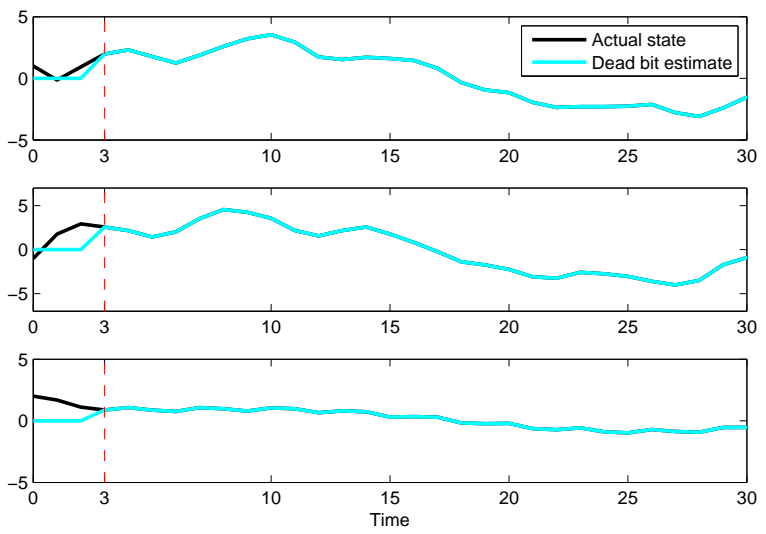

Fig. 1. Real state and dead bit estimate for $h=3$.
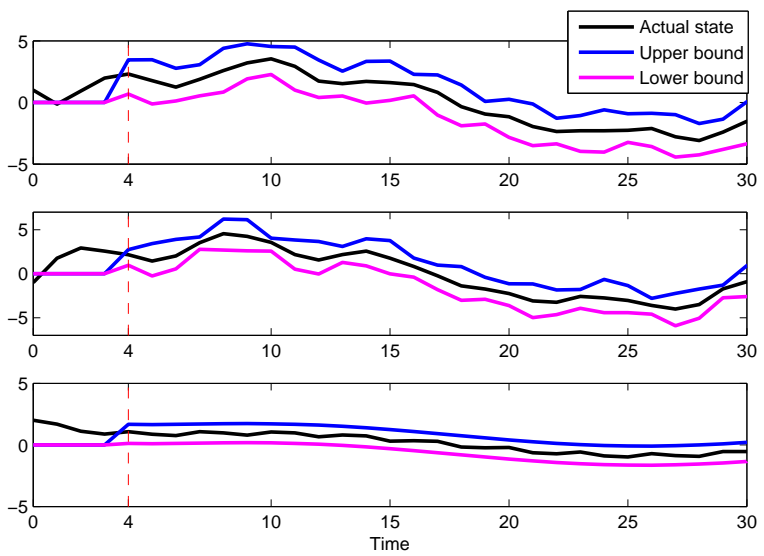

Fig. 2. Fixed-time interval estimation with upper and lower bounds for $h=4$.

\section{CONCLUSION}

Based on the use of past values of the input and the output of the studied system, a new technique of design of fixedtime observers for a family of discrete-time nonlinear timevarying systems is presented. The disturbances on the state as well as on the measurement are both taken into account. It is worth highlighting that neither information on the bound of the initial conditions nor direct or indirect notion of nonnegative and cooperative system are needed in our development. We provide exact values of the solutions in the absence of uncertainties and intervals when the uncertainties are present after a fixed time. Finally, an illustrative example highlights the efficiency of our methodology.

\section{REFERENCES}

[1] G. Besancon, Nonlinear Observers and Applications, Springer, 2007.

[2] F. Cacace, A. Germani, C. Manes, A New Approach to Design Interval Observers for Linear Systems, IEEE Transactions on Automatic Control, 60, pp. 1665 - 1670, 2015.

[3] K. Chaib-Draa, A. Zemouche, R. Rajamani, T.M. Laleg-Kirati, Finitetime state estimation of discrete-time linear systems with some extensions. Application to steering lateral vehicle model, IEEE 58th Conference on Decision and Control, Nice, France, to appear, 2019

[4] T.N. Dinh, F. Mazenc, T. Raïssi, Finite-time guaranteed state estimation for discrete-time systems with disturbances, 4th conference on Control and Fault-Tolerant Systems, Casablanca, Morocco, pp. 342347, 2019

[5] D. Efimov, W. Perruquetti, T. Raïssi, A. Zolghadri, Interval Observers for Time-Varying Discrete-Time Systems, IEEE Transactions on Automatic Control, 58, pp. 3218-3224, 2013.

[6] R. Engel, G. Kreisselmeier, A Continuous-Time Observer Which Converges in Finite Time, IEEE Transactions on Automatic Control, 47, pp. 1202-1204, 2002

[7] J. L. Gouzé, A. Rapaport, M. Z. Hadj-Sadok, Interval Observers for Uncertain Biological Systems, Ecological Modelling, 133, pp. 45-56, 2000

[8] M. S. Grewal, A. P. Andrews, Kalman Filtering: Theory and Practice Using MATLAB (2nd Edition), John Wiley \& Sons, 2001.

[9] F. Lopez-Ramirez, A. Polyakov, D. Efimov, W. Perruquetti, FiniteTime and Fixed-Time Observers Design via Implicit Lyapunov Function, 2016 European Control Conference (ECC), pp. 289-294, 2016.

[10] F. Mazenc, T. N. Dinh, S.-I. Niculescu, Robust Interval Observers and Stabilization Design for Discrete-time Systems with Input and Output, Automatica, 49, pp. 3490-3497, 2013.

[11] F. Mazenc, T. N. Dinh, S.-I. Niculescu, Interval Observers For Discrete-time Systems, International Journal of Robust and Nonlinear Control, 24, pp. 2867-2890, 2014.

[12] F. Mazenc, E. Fridman, W. Djema, Estimation of solutions of observable nonliear systems with disturbances, Systems \& Control Letters, 79, pp. 47-58, 2015.

[13] T. Menard, E. Moulay, W. Perruquetti, Fixed-time Observer with Simple Gains for Uncertain Systems, Automatica, 81, pp. 438-446, 2017.

[14] T. Raïssi, D. Efimov, Some recent results on the design and implementation of interval observers for uncertain systems, atAutomatisierungstechnik, 66, pp. 213-224, 2018.

[15] H. Rios, A.R. Teel, A Hybrid Fixed-time Observer for State Estimation of Linear Systems, Automatica, 87, pp. 103-112, 2018.

[16] Z. Wang, C.-C. Lim, Y. Shen, Interval Observer Design for Uncertain Discrete-time Linear Systems, Systems \& Control Letters, 116, pp. 4146, 2018

[17] J. Zhang, M. Chadli, Y. Wang, A fixed-time observer for discretetime singular systems with unknown inputs, Applied Mathematics and Computation, 363, pp. 1-12, 2019.

[18] M. Zhong, D. Zhou, S.X. Ding, On Designing $H_{\infty}$ Fault Detection Filter for Linear Discrete Time-Varying Systems, IEEE Transactions on Automatic Control, 55, pp. 1689-1695, 2010. 